\title{
PENULTIMATE APPROXIMATION FOR THE DISTRIBUTION OF THE EXCESSES
}

\author{
RYM WORMS ${ }^{1}$
}

\begin{abstract}
Let $F$ be a distribution function (d.f) in the domain of attraction of an extreme value distribution $H_{\gamma}$; it is well-known that $F_{u}(x)$, where $F_{u}$ is the d.f of the excesses over $u$, converges, when $u$ tends to $s_{+}(F)$, the end-point of $F$, to $G_{\gamma}\left(\frac{x}{\sigma(u)}\right)$, where $G_{\gamma}$ is the d.f. of the Generalized Pareto Distribution. We provide conditions that ensure that there exists, for $\gamma>-1$, a function $\Lambda$ which verifies $\lim _{u \rightarrow s_{+}(F)} \Lambda(u)=\gamma$ and is such that $\Delta(u)=\sup _{x \in\left[0, s_{+}(F)-u[\right.}\left|\bar{F}_{u}(x)-\bar{G}_{\Lambda(u)}(x / \sigma(u))\right|$ converges to 0 faster than $d(u)=\sup _{x \in\left[0, s_{+}(F)-u[\right.}\left|\bar{F}_{u}(x)-\bar{G}_{\gamma}(x / \sigma(u))\right|$.
\end{abstract}

Mathematics Subject Classification. 60G70, 62G20.

Received February 26, 2001. Revised October 5, 2001.

\section{INTRODUCTION}

Let $F$ be a distribution function in the domain of attraction of an extreme value distribution $H_{\gamma}$, where

$$
\begin{aligned}
& H_{\gamma}(x)=\exp \left(-(1+\gamma x)^{-1 / \gamma}\right) \text { for } x \text { such that } 1+\gamma x>0 \text {, if } \gamma \neq 0 \text {, } \\
& =\exp \left(-\mathrm{e}^{-x}\right) \quad \text { for } x \in \mathbb{R} \quad \text { if } \gamma=0,
\end{aligned}
$$

which means there exist sequences $\left(\alpha_{n}\right)$ and $\left(\sigma_{n}\right)$ such that

$$
\hat{d}_{n}=\sup _{x \in \mathbb{R}}\left|F^{n}(x)-H_{\gamma}\left(\frac{x-\alpha_{n}}{\sigma_{n}}\right)\right| \longrightarrow 0, \text { as } n \rightarrow+\infty \text {. }
$$

It is well-known (see [8] and [1]) that this is equivalent to the existence of $\sigma(u)>0$ such that

$$
d(u)=\sup _{x \in\left[0, s_{+}(F)-u[\right.}\left|\bar{F}_{u}(x)-\bar{G}_{\gamma}\left(\frac{x}{\sigma(u)}\right)\right| \longrightarrow 0, \text { as } u \rightarrow s_{+}(F),
$$

where $s_{+}(F)=\sup \{x, F(x)<1\}$ is the upper endpoint of $F$ (and $s_{-}(F)=\inf \{x, F(x)>0\}$ its lower endpoint), $\bar{F}_{u}(x)(=\bar{F}(x+u) / \bar{F}(u)=(1-F(x+u)) /(1-F(u)))$ is the survival function of the excess over $u$ and $\bar{G}_{\gamma}$ the

Keywords and phrases: Generalized Pareto Distribution, excesses, penultimate approximation, rate of convergence.

${ }^{1}$ Université de Marne-la-Vallée, Équipe d'Analyse et de Mathématiques Appliquées, 5 boulevard Descartes, Champs-sur-Marne, 77454 Marne-la-Vallee Cedex 2, France; e-mail: ramdani@math.univ-mlv.fr 
survival function of the Generalized Pareto Distribution, with

$$
\begin{aligned}
& \bar{G}_{\gamma}(x)=(1+\gamma x)^{-1 / \gamma} \text { for } x \geq 0 \text { such that } 1+\gamma x>0 \text {, if } \gamma \neq 0 \text {, } \\
& =\mathrm{e}^{-x} \quad \text { for } x \in \mathbb{R}_{+} \quad \text { if } \gamma=0 .
\end{aligned}
$$

$H_{\gamma}$ will be called in this paper the "ultimate" approximation of $F^{n}$ and, similarly, $\bar{G}_{\gamma}$ the "ultimate" approximation of $\bar{F}_{u}$. In 1928, Fisher and Tippet [4] showed empirically, for the normal distribution (for which $\gamma=0$ ), that there exists a sequence of extreme value distributions $H_{\gamma_{n}}$ of Weibull type $\left(\gamma_{n}<0\right.$ and $\left.\gamma_{n} \rightarrow 0\right)$, which is a better approximation of $F^{n}$ than its limiting distribution $H_{\gamma}$. They called $H_{\gamma_{n}}$ a "penultimate" approximation of $F^{n}$.

Cohen, in [3], studied the case of the Gumbel domain of attraction $(\gamma=0)$. He exhibited a penultimate approximation of $F^{n}$, for distributions in a sub-class of this domain and compared the rates of convergence to 0 of

$$
\hat{d}_{n}=\sup _{x \in \mathbb{R}}\left|F^{n}\left(\sigma_{n} x+\alpha_{n}\right)-H_{\gamma}(x)\right|
$$

and

$$
\hat{\Delta}_{n}=\sup _{x \in \mathbb{R}}\left|F^{n}\left(\sigma_{n} x+\alpha_{n}\right)-H_{\gamma_{n}}(x)\right|,
$$

for appropriate normalizing sequences $\alpha_{n}$ and $\sigma_{n}$.

In [5] and [7], Gomes studied the rates of convergence of $\hat{d}_{n}$ and $\hat{\Delta}_{n}$, in the case $\gamma=0$ and then in the other cases $(\gamma>0$ and $\gamma<0)$.

More recently, Gomes and de Haan, in [6], gave a necessary condition for the existence of a penultimate approximation for $F^{n}$. In other words, they provided a condition for the existence of a sequence $\gamma_{n}$ tending to $\gamma$ such that the rate of convergence to 0 of $\hat{\Delta}_{n}$ is better than the rate of $\hat{d}_{n}$.

Now, regarding the Generalized Pareto approximation for the distribution of the excesses, we have studied, in [9], the rate of convergence to 0 of

$$
d(u)=\sup _{x \in\left[0, s_{+}(F)-u[\right.}\left|\bar{F}_{u}(x)-\bar{G}_{\gamma}\left(\frac{x+u-\alpha(u)}{\sigma(u)}\right)\right|,
$$

for appropriate normalizing functions $\alpha$ and $\sigma$.

The aim of this paper is to study the existence of a penultimate approximation for this distribution of the excesses. In other words, we look for conditions under which there exists a function $\Lambda$ such that $\Lambda(u) \longrightarrow \gamma$, as $u \rightarrow s_{+}(F)$, and the rate of convergence to 0 of

$$
\Delta(u)=\sup _{x \in\left[0, s_{+}(F)-u[\right.}\left|\bar{F}_{u}(x)-\bar{G}_{\Lambda(u)}\left(\frac{x+u-\alpha(u)}{\sigma(u)}\right)\right|
$$

is better than the rate of $d(u)$, for appropriate normalizing functions $\alpha$ and $\sigma$.

In Section 1, we present the framework. In Section 2, we provide a necessary condition for the existence of a penultimate approximation, with the appropriate normalizing functions. In Section 3, we state our results. In Section 4, we prove the main result.

\section{Assumptions And PRELIMinary PROPERTIES}

In the sequel, we suppose that $F$ is four times differentiable and that its inverse $F^{-1}$ exists. We define the mapping $V$ from $\mathbb{R}_{+}^{*}$ onto $] s_{-}(F), s_{+}(F)\left[\right.$, by $V(t)=\bar{F}^{-1}\left(\mathrm{e}^{-t}\right)=\bar{F}^{-1}\left(\bar{G}_{0}(t)\right)$ and we note $A(t)=\frac{V^{\prime \prime}(\ln t)}{V^{\prime}(\ln t)}-\gamma$. 
We have established, in [9] (and [10]), the rate of convergence to 0 of $d(u)$, under the following first and second order conditions:

$$
\lim _{t \rightarrow+\infty} A(t)=0
$$

and

$$
A \text { is of constant sign at }+\infty \text { and there exists } \rho \leq 0 \text { such that }|A| \in R V_{\rho}{ }^{2}
$$

Here, we use a second order condition which (see [2]) is stronger than (4):

$$
A \text { is of constant sign at }+\infty \text { and there exists } \rho \leq 0 \text { such that } \lim _{t \rightarrow+\infty} \frac{t A^{\prime}(t)}{A(t)}=\rho .
$$

We shall see in the following section that, under assumptions (3) and (4), a necessary condition for the existence of a penultimate approximation for the distribution of the excesses is $\rho=0$. In order to compute the rate of convergence of $\Delta(u)$, we introduce the following condition:

$$
A^{\prime} \text { is of constant sign at }+\infty \text { and } \lim _{t \rightarrow+\infty} \frac{t A^{\prime \prime}(t)}{A^{\prime}(t)}=-1 \text {, }
$$

which, by an immediate application of the l'Hospital rule, implies $\rho=0$.

Remark 1. Gomes and de Haan, in [6], make use of conditions (3), (5) and (6), where $A$ is replaced by $\hat{A}(t)=\frac{\hat{V}^{\prime \prime}(\ln t)}{\hat{V}^{\prime}(\ln t)}-\gamma$, with $\hat{V}(t)=F^{-1}\left(\exp \left(-\mathrm{e}^{-t}\right)\right)$.

The results stated in the following proposition using functions $V$ and $A$ are stated in [6] (see 1.14 and Lem. 2.1) using functions $\hat{V}$ and $\hat{A}$. This proposition has to be compared with Proposition 1 in [9] (or Prop. 1 in $[10])$.

Proposition 1. Under conditions (3), (5) and (6), for $\gamma \in \mathbb{R}$,

(i)

$$
\lim _{t \rightarrow+\infty}\left[\frac{V(t+x)-V(t)}{V^{\prime}(t)}-\frac{\mathrm{e}^{\lambda(t) x}-1}{\lambda(t)}\right] / \lambda^{\prime}(t)=M_{\gamma}(x),
$$

where

$$
M_{\gamma}(x)=\frac{1}{2} \int_{0}^{x} u^{2} \mathrm{e}^{\gamma u} \mathrm{~d} u
$$

(ii) $\forall \epsilon>0, \exists t_{0} \geq 0, \forall t \geq t_{0}, \forall x \geq t_{0}-t$,

- if $x \geq 0$,

$$
(1-\epsilon) \mathrm{e}^{-\epsilon x} M_{\gamma}(x)<\frac{\frac{V(t+x)-V(t)}{V^{\prime}(t)}-\frac{\mathrm{e}^{\lambda(t) x}-1}{\lambda(t)}}{\lambda^{\prime}(t)}<(1+\epsilon) \mathrm{e}^{\epsilon x} M_{\gamma}(x),
$$

- if $x \leq 0$,

$$
(1+\epsilon) \mathrm{e}^{-\epsilon x} M_{\gamma}(x)<\frac{\frac{V(t+x)-V(t)}{V^{\prime}(t)}-\frac{\mathrm{e}^{\lambda(t) x}-1}{\lambda(t)}}{\lambda^{\prime}(t)}<(1-\epsilon) \mathrm{e}^{\epsilon x} M_{\gamma}(x) .
$$

\footnotetext{
${ }^{2} f \in R V_{\alpha}$ (i.e. $f$ is regularly varying with index $\alpha$ ) means that for all $x>0, \lim _{t \rightarrow+\infty} \frac{f(t x)}{f(t)}=x^{\alpha}$ (see [2]).
} 
Proof. We prove only (i) (for (ii), see Lem. 2.1 in [6]).

$$
\begin{aligned}
\frac{V(t+x)-V(t)}{V^{\prime}(t)}-\frac{\mathrm{e}^{\lambda(t) x}-1}{\lambda(t)} & =\int_{0}^{x} \frac{V^{\prime}(t+s)}{V^{\prime}(t)} \mathrm{d} s-\int_{0}^{x} \exp \left(s \frac{V^{\prime \prime}(t)}{V^{\prime}(t)}\right) \mathrm{d} s \\
& =\int_{0}^{x} \exp \left(s \frac{V^{\prime \prime}(t)}{V^{\prime}(t)}\right)\left[\exp \left(\ln V^{\prime}(t+s)-\ln V^{\prime}(t)-s \frac{V^{\prime \prime}(t)}{V^{\prime}(t)}\right)-1\right] \mathrm{d} s \\
& =\int_{0}^{x} \exp \left(s \frac{V^{\prime \prime}(t)}{V^{\prime}(t)}\right)\left[\exp \left(\int_{0}^{s}\left(\frac{V^{\prime \prime}(t+z)}{V^{\prime}(t+z)}-\frac{V^{\prime \prime}(t)}{V^{\prime}(t)}\right) \mathrm{d} z\right)-1\right] \mathrm{d} s
\end{aligned}
$$

Now $\frac{V^{\prime \prime}(t)}{V^{\prime}(t)}=\lambda(t)$ and $\frac{V^{\prime \prime}(t+z)}{V^{\prime}(t+z)}-\frac{V^{\prime \prime}(t)}{V^{\prime}(t)}=\int_{0}^{z} \lambda^{\prime}(t+q) \mathrm{d} q$, hence

$$
\left[\frac{V(t+x)-V(t)}{V^{\prime}(t)}-\frac{\mathrm{e}^{\lambda(t) x}-1}{\lambda(t)}\right] / \lambda^{\prime}(t)=\int_{0}^{x} \exp (s \lambda(t))\left[\frac{\exp \left(\lambda^{\prime}(t) \int_{0}^{s} \int_{0}^{z} \frac{\lambda^{\prime}(t+q)}{\lambda^{\prime}(t)} \mathrm{d} q \mathrm{~d} z\right)-1}{\lambda^{\prime}(t)}\right] \mathrm{d} s
$$

When $t \rightarrow+\infty, \lambda(t) \longrightarrow \gamma($ from $(3)), \lambda^{\prime}(t) \longrightarrow 0$ (from (5)), and $\frac{\lambda^{\prime}(t+q)}{\lambda^{\prime}(t)} \longrightarrow 1$ uniformly on every interval $[0, X]$ (from Lem. 1). Hence, for all $x \in \mathbb{R}$,

$$
\left[\frac{V(t+x)-V(t)}{V^{\prime}(t)}-\frac{\mathrm{e}^{\lambda(t) x}-1}{\lambda(t)}\right] / \lambda^{\prime}(t) \longrightarrow \int_{0}^{x} \mathrm{e}^{\gamma s} \frac{s^{2}}{2} \mathrm{~d} s=M_{\gamma}(x)
$$

Remark 2. Note that $M_{\gamma}(0)=0, M_{\gamma}$ is positive on $\mathbb{R}_{+}^{*}$ and negative on $\mathbb{R}_{-}^{*}$.

\section{NeCESSARY CONDITION FOR PENULtimate APPROXIMATION}

Our aim is to find conditions under which there exists a function $\Lambda$ such that $\Lambda(u) \longrightarrow \gamma$, as $u \rightarrow s_{+}(F)$, and the rate of convergence to 0 of

$$
\begin{aligned}
\Delta(u) & =\sup \left(\left|\bar{F}_{u}(x)-\bar{G}_{\Lambda(u)}\left(\frac{x+u-\alpha(u)}{\sigma(u)}\right)\right| ; x \in\left[0, s_{+}(F)-u[)\right.\right. \\
& =\sup \left(\left|\bar{F}_{u}(\sigma(u) x-u+\alpha(u))-\bar{G}_{\Lambda(u)}(x)\right| ; x \in\left[\frac{u-\alpha(u)}{\sigma(u)}, \frac{s_{+}(F)-\alpha(u)}{\sigma(u)}[)\right.\right.
\end{aligned}
$$

is faster than $A\left(\mathrm{e}^{V^{-1}(u)}\right)$, which was the rate in the ultimate case (see [9] or [10]). A method for this is to $\operatorname{express}^{3} \bar{F}_{u}(x)-\bar{G}_{\Lambda(u)}\left(\frac{x+u-\alpha(u)}{\sigma(u)}\right)$ using $\bar{G}_{0}$ (i.e. $\left.x \mapsto \mathbb{I}_{]-\infty, 0[}(x)+\mathbb{I}_{[0,+\infty[}(x) \mathrm{e}^{-x}\right)$.

For this purpose, we introduce the function $\phi_{u}$, defined from $\left[0, s_{+}(F)-u\left[\right.\right.$ onto $\mathbb{R}_{+}$, by

$$
\phi_{u}(x)=V^{-1}(x+u)-V^{-1}(u) .
$$

We obtain

$$
\bar{F}_{u}(x)=\bar{G}_{0}\left(\phi_{u}(x)\right)
$$

\footnotetext{
${ }^{3}$ As we did in [9] or [10] in order to study $\mathrm{d}(u)$.
} 
We note that, for $\gamma \neq 0$ and $x \in \mathbb{R}_{+}$,

$$
\bar{G}_{\gamma}(x)=\bar{G}_{0}\left(\frac{1}{\gamma} \ln (1+\gamma x)\right)
$$

If $\Lambda(u) \neq 0$, for $u$ sufficiently large ${ }^{4}$, it follows that, for all $x \in\left[0, s_{+}(F)-u[\right.$,

$$
\bar{F}_{u}(x)-\bar{G}_{\Lambda(u)}\left(\frac{x+u-\alpha(u)}{\sigma(u)}\right)=\bar{G}_{0}\left(\phi_{u}(x)\right)-\bar{G}_{0}\left(\frac{1}{\Lambda(u)} \ln \left(1+\Lambda(u) \frac{V\left(\phi_{u}(x)+V^{-1}(u)\right)-\alpha(u)}{\sigma(u)}\right)\right) .
$$

Then we can write

$$
\Delta(u)=\sup _{s \in \mathbb{R}_{+}}\left|B_{u}(s)\right|,
$$

where

$$
B_{u}(s)=\bar{G}_{0}(s)-\bar{G}_{0}\left(s+q_{u}(s)\right)
$$

and

$$
q_{u}(s)=\frac{1}{\Lambda(u)} \ln \left(1+\Lambda(u) \frac{V\left(s+V^{-1}(u)\right)-\alpha(u)}{\sigma(u)}\right)-s
$$

Now, let us choose

$$
\sigma(u)=V^{\prime}\left(V^{-1}(u)\right) \text { and } \alpha(u)=V\left(V^{-1}(u)\right)=u \text {. }
$$

These are the normalizing functions used in the ultimate case for $\gamma>-1$ (see [9] or [10]).

Theorem 1. Under (3) and (4), if $\alpha(u)$ and $\sigma(u)$ are as in (12), a necessary condition to have, for all $\gamma \in \mathbb{R}$ and all $s \in \mathbb{R}_{+}$,

$$
B_{u}(s)=\bar{G}_{0}(s)-\bar{G}_{0}\left(\frac{1}{\Lambda(u)} \ln \left\{1+\Lambda(u) \frac{V\left(s+V^{-1}(u)\right)-\alpha(u)}{\sigma(u)}\right\}\right)=o\left(A\left(\mathrm{e}^{V^{-1}(u)}\right)\right)
$$

is: $\rho=0$ and $\Lambda(u)=\gamma+A\left(\mathrm{e}^{V^{-1}(u)}\right)$.

Proof. The idea of the proof is due to Gomes and de Haan (see [6]).

Let $\bar{G}_{0}^{\prime}(0)$ be the right derivative of $\bar{G}_{0}$ at 0 . As $\frac{1}{\Lambda(u)} \ln \left\{1+\Lambda(u) \frac{V\left(s+V^{-1}(u)\right)-u}{V^{\prime}\left(V^{-1}(u)\right)}\right\} \geq 0$, for all $s \in \mathbb{R}_{+}$,

$$
\bar{G}_{0}(s)-\bar{G}_{0}\left(\frac{1}{\Lambda(u)} \ln \left\{1+\Lambda(u) \frac{V\left(s+V^{-1}(u)\right)-\alpha(u)}{\sigma(u)}\right\}\right)=\bar{G}_{0}^{\prime}(s)\left(s-J_{u}(s)\right)+O\left(s-J_{u}(s)\right)^{2}
$$

\footnotetext{
large.

${ }^{4}$ If $\gamma \neq 0$, this is obvious because $\Lambda(u)$ tends to $\gamma$, but if $\gamma=0$, we have to suppose that $\Lambda(u)$ is of constant sign for $u$ sufficiently
} 
where

$$
J_{u}(s)=\frac{1}{\gamma+z_{u}} \ln \left(1+K_{u}\left(z_{u}\right)\right)
$$

with $z_{u}=\Lambda(u)-\gamma$ and $K_{u}\left(z_{u}\right)=\left(\gamma+z_{u}\right) \frac{V\left(s+V^{-1}(u)\right)-u}{V^{\prime}\left(V^{-1}(u)\right)}$.

We know from [9] (or [10]) that conditions (3) and (4) imply that for all $\gamma \in \mathbb{R}$ and all $x \in \mathbb{R}$,

$$
\left[\frac{V(t+s)-V(t)}{V^{\prime}(t)}-\int_{0}^{s} \mathrm{e}^{\gamma x} \mathrm{~d} x\right] / A\left(\mathrm{e}^{t}\right) \stackrel{t \rightarrow+\infty}{\longrightarrow} \int_{0}^{s} \mathrm{e}^{\gamma x} \int_{0}^{x} \mathrm{e}^{\rho z} \mathrm{~d} z \mathrm{~d} x=I_{\gamma, \rho}(s)
$$

From now on, we treat only the case $\gamma \neq 0$ (the case $\gamma=0$ is similar).

$$
\begin{aligned}
1+K_{u}\left(z_{u}\right) & =1+\left(\gamma+z_{u}\right)\left(\frac{\mathrm{e}^{\gamma s}-1}{\gamma}+A\left(\mathrm{e}^{V^{-1}(u)}\right) I_{\gamma, \rho}(s)+o\left(A\left(\mathrm{e}^{V^{-1}(u)}\right)\right)\right) \\
& =\mathrm{e}^{\gamma s}+\frac{\mathrm{e}^{\gamma s}-1}{\gamma} z_{u}+\gamma A\left(\mathrm{e}^{V^{-1}(u)}\right) I_{\gamma, \rho}(s)+o\left(A\left(\mathrm{e}^{V^{-1}(u)}\right)\right)
\end{aligned}
$$

Then

$$
\ln \left(1+K_{u}\left(z_{u}\right)\right)=\gamma s+\frac{1-\mathrm{e}^{-\gamma s}}{\gamma} z_{u}+\gamma A\left(\mathrm{e}^{V^{-1}(u)}\right) \mathrm{e}^{-\gamma s} I_{\gamma, \rho}(s)+o\left(z_{u}\right)+o\left(A\left(\mathrm{e}^{V^{-1}(u)}\right)\right)
$$

and

$$
J_{u}(s)=\frac{1}{\gamma}\left(1-z_{u} / \gamma+o\left(z_{u}\right)\right)\left(\gamma s+\frac{1-\mathrm{e}^{-\gamma s}}{\gamma} z_{u}+\gamma A\left(\mathrm{e}^{V^{-1}(u)}\right) \mathrm{e}^{-\gamma s} I_{\gamma, \rho}(s)+o\left(z_{u}\right)+o\left(A\left(\mathrm{e}^{V^{-1}(u)}\right)\right)\right)
$$

whence

$$
s-J_{u}(s)=\frac{1}{\gamma^{2}}\left(\gamma s-1+\mathrm{e}^{-\gamma s}\right) z_{u}-A\left(\mathrm{e}^{V^{-1}(u)}\right) \mathrm{e}^{-\gamma s} I_{\gamma, \rho}(s)+o\left(z_{u}\right)+o\left(A\left(\mathrm{e}^{V^{-1}(u)}\right)\right) .
$$

The rate of convergence of $B_{u}(s)$ will be better than $O\left(A\left(\mathrm{e}^{V^{-1}(u)}\right)\right)$ (the rate in the ultimate case), for all $s \in \mathbb{R}_{+}$, if we can choose $z_{u}$ tending to 0 as $u \rightarrow s_{+}(F)$, such that, for all $s \in \mathbb{R}_{+}$,

$$
\left.\frac{1}{\gamma^{2}}\left(-\gamma s+1-\mathrm{e}^{-\gamma s}\right) z_{u}+A\left(\mathrm{e}^{V^{-1}(u)}\right)\right) \mathrm{e}^{-\gamma s} I_{\gamma, \rho}(s)=0
$$

and $z_{u}=O\left(A\left(\mathrm{e}^{V^{-1}(u)}\right)\right)$.

This is possible only if $\rho=0$. Indeed, $I_{\gamma, 0}(s)=\int_{0}^{s} y \mathrm{e}^{\gamma y} \mathrm{~d} y=-\frac{\mathrm{e}^{\gamma s}}{\gamma^{2}}\left(-\gamma s+1+\mathrm{e}^{-\gamma s}\right)$ and then it suffices to choose $z_{u}=A\left(\mathrm{e}^{V^{-1}(u)}\right)$.

Remark 3. We know from [9] (or [10]) that another choice of normalizing functions $\left(\alpha^{*}(u), \sigma^{*}(u)\right)$ is possible for $\gamma<0$. However, one can check that the same kind of computations as above shows that this choice does not lead to a penultimate approximation.

\section{Rates of CONVERGENCE}

In order to determine the rate of convergence to 0 of

$$
\begin{aligned}
\Delta(u) & =\sup \left(\left|\bar{F}_{u}(x)-\bar{G}_{\Lambda(u)}\left(\frac{x+u-\alpha(u)}{\sigma(u)}\right)\right| ; x \in\left[0, s_{+}(F)-u[)\right.\right. \\
& =\sup _{s \in \mathbb{R}_{+}}\left|B_{u}(s)\right|,
\end{aligned}
$$


where $B_{u}(s)$ is defined in (10), we begin (Th. 2, whose proof is given in Sect. 4) by giving the rate of uniform convergence to 0 of $B_{u}(s)$, as $u$ tends to $s_{+}(F)$, where the normalizing functions are chosen as in (12) and $\Lambda$ is defined by $\Lambda(u)=\gamma+A\left(\mathrm{e}^{V^{-1}(u)}\right)$, as in Theorem 1 .

Theorem 2. Let $F$ be a distribution function satisfying (3), (5) and (6). Define $C_{\gamma}$ by

$$
C_{\gamma}(s)=\mathrm{e}^{-\gamma s}\left|\bar{G}_{0}^{\prime}(s)\right| M_{\gamma}(s)=\exp [-(1+\gamma) s] M_{\gamma}(s) .
$$

Then, for $\gamma>-1, \frac{B_{u}}{\mathrm{e}^{V^{-1}(u)} A^{\prime}\left(\mathrm{e}^{V^{-1}(u)}\right)}$ converges to $C_{\gamma}$, uniformly on $\mathbb{R}_{+}$, as $u$ tends to $s_{+}(F)$.

The rate of convergence of $\Delta(u)$ to 0 follows straightforwardly:

Corollary 1. Under the same hypothesis and notations as Theorem 2, if $\gamma>-1$, as $u$ tends to $s_{+}(F)$,

$$
\Delta(u)=\sup _{x \in\left[0, s_{+}(F)-u[\right.}\left|\bar{F}_{u}(x)-\bar{G}_{\Lambda(u)}\left(\frac{x+u-\alpha(u)}{\sigma(u)}\right)\right|=O\left(\mathrm{e}^{V^{-1}(u)} A^{\prime}\left(\mathrm{e}^{V^{-1}(u)}\right)\right) .
$$

Remark 4. Note that $\mathrm{e}^{V^{-1}(u)}=1 / \bar{F}(u)$.

Remark 5. The condition $\lim _{t \rightarrow+\infty} \frac{t A^{\prime}(t)}{A(t)}=0$ (see (5)) ensures that the rate of convergence to 0 of $\Delta(u)$ is better than the rate of $d(u)$, which is of order $A\left(\mathrm{e}^{V^{-1}(u)}\right)$ (see Cor. 1 in [9]). However, it follows from Lemma 1 below that the rate of convergence in the penultimate case remains a slowly varying function $\left(\in R V_{0}\right)$.

Lemma 1. If we note

$$
\lambda(t)=\frac{V^{\prime \prime}(t)}{V^{\prime}(t)}=A\left(\mathrm{e}^{t}\right)+\gamma
$$

then, under (6), for all $q$ in $\mathbb{R}$,

$$
\lim _{t \rightarrow+\infty} \frac{\lambda^{\prime}(t+q)}{\lambda^{\prime}(t)}=1
$$

In other words, $t \mapsto\left|\lambda^{\prime}(\ln t)\right|=\left|t A^{\prime}(t)\right|$ is $R V_{0}$. This convergence is uniform on every compact set of the form $[0, T]$.

Proof. For $q$ in $\mathbb{R}$ and $t \geq \max (0,-q)$,

$$
\ln \frac{\lambda^{\prime}(t+q)}{\lambda^{\prime}(t)}=\ln \left|\lambda^{\prime}(t+q)\right|-\ln \left|\lambda^{\prime}(t)\right|=\int_{t}^{t+q} \frac{\lambda^{\prime \prime}(r)}{\lambda^{\prime}(r)} \mathrm{d} r
$$

Condition (6) implies that $\frac{\lambda^{\prime \prime}(t)}{\lambda^{\prime}(t)} \longrightarrow 0$, as $u \rightarrow s_{+}(F)$. It follows that $\ln \frac{\lambda^{\prime}(t+q)}{\lambda^{\prime}(t)}$ tends to 0 uniformly on every compact set $[0, T]$. Hence $\frac{\lambda^{\prime}(t+q)}{\lambda^{\prime}(t)}$ tends to 1 uniformly on $[0, T]$.

As a consequence of Theorem 2, we can also give the uniform rate of convergence to 0 of $\bar{F}_{u}(\sigma(u) y)-\bar{G}_{\Lambda(u)}$ :

Theorem 3. Under the same hypothesis and notations as in Theorem 2, if $\gamma>-1$,

$$
\frac{\bar{F}_{u}\left(V^{\prime}\left(V^{-1}(u)\right) y\right)-\bar{G}_{\Lambda(u)}(y)}{\mathrm{e}^{V^{-1}(u)} A^{\prime}\left(\mathrm{e}^{V^{-1}(u)}\right)},
$$


converges, when $u \rightarrow s_{+}(F)$, to

$$
\begin{array}{ll}
D_{\gamma}(y)=C_{\gamma}\left(\frac{1}{\gamma} \ln (1+\gamma y)\right) & \text { if } \gamma \neq 0, \\
D_{0}(y)=C_{0}(y) & \text { if } \gamma=0 .
\end{array}
$$

This convergence is uniform:

- on $\mathbb{R}_{+}$if $s_{+}(F)=+\infty$ (particularly if $\left.\gamma>0\right)$;

- $[0, X]$ (where $X>0)$ if $\gamma=0$ and $s_{+}(F)<+\infty$;

- $[0, X]$ (where $\left.0<X<-\frac{1}{\gamma}\right)$, if $\gamma<0$.

The proof of this theorem is similar to the proof of theorem 3 in [9] (or Th. 4 in [10]). The only difference is that, if we define $\psi_{u}$ by $s=\psi_{u}(y)=V^{-1}(\sigma(u) y+\alpha(u))-V^{-1}(u)$, an important step is to prove the uniform convergence to 0 of $\bar{G}_{0}\left(\psi_{u}(y)\right)-\bar{G}_{\gamma}(y)$, on $\left[0, s_{+, \gamma}\left[\right.\right.$ (where $s_{+, \gamma}$ is the upper end point of $\bar{G}_{\gamma}$ ); here, Theorem 2 ensures the uniform convergence to 0 of $\bar{G}_{0}\left(\psi_{u}(y)\right)-\bar{G}_{\Lambda(u)}(y)$. Besides, using the fact that $\bar{G}_{\gamma}$ is decreasing and that $\lim _{y \rightarrow s_{+}(F)} \bar{G}_{\gamma}(y)=0$, we prove that $\bar{G}_{\Lambda(u)}(y)-\bar{G}_{\gamma}(y)$ converges to 0 , uniformly on $\left[0, s_{+, \gamma}[\right.$.

Example 1. Distribution functions defined by

$$
1-F(x)=\exp \left(-x^{\beta} \tilde{l}(x)\right)
$$

where $\beta>0$ and $\tilde{l}$ is a smooth slowly varying function ${ }^{5}$, are called of Weibull type. They are in the Gumbel domain of attraction. For these distributions, if $\beta \notin\left\{\frac{1}{2}, 1\right\}$,

$$
A(t)=\frac{V^{\prime \prime}(\ln t)}{V^{\prime}(\ln t)} \sim\left(\frac{1}{\beta}-1\right) \frac{1}{\ln t}, \text { and } t A^{\prime}(t) \sim\left(1-\frac{1}{\beta}\right) \frac{1}{(\ln t)^{2}} \text {, as } t \rightarrow+\infty
$$

Assumptions of Theorem 2 are satisfied. It follows that we can get a penultimate approximation for this type of distributions, as the Normal distribution $(\beta=2)$ and the Weibull distribution $(\beta>0$ and $\tilde{l}=1)$.

We deduce from (14) that the rate of convergence is of order $\frac{1}{-\ln (1-F(u))}$ in the ultimate case and $\frac{1}{(-\ln (1-F(u)))^{2}}$ in the penultimate one.

\section{Proof of Theorem 2}

The proof of Theorem 2 follows the same lines as the proof of Theorem 1 in [9] (the ultimate case). Here are the two main steps of the proof:

(i) We find a positive function $S$ satisfying $S(u) \rightarrow+\infty$ and such that, when $u \rightarrow s_{+}(F)$,

$$
\frac{B_{u}}{\mathrm{e}^{V^{-1}(u)} A^{\prime}\left(\mathrm{e}^{V^{-1}(u)}\right)} \text { converges to } C_{\gamma}
$$

uniformly on $[0, S(u)]$, and

$$
\frac{1}{\mathrm{e}^{V^{-1}(u)} A^{\prime}\left(\mathrm{e}^{V^{-1}(u)}\right)} \bar{G}_{0}(S(u)) \text { converges to } 0 .
$$

(ii) We extend the convergence established in (i) to $\mathbb{R}_{+}$.

\footnotetext{
${ }^{5}$ This means that $\tilde{l}$ is $C^{\infty}$ and is slowly varying.
} 
- We begin by showing how (ii) is derived from (i). As $\lim _{x \rightarrow+\infty} C_{\gamma}(x)=0$, for $\gamma>-1$, it is sufficient to show that $\sup _{s \geq S(u)} \frac{B_{u}(s)}{\mathrm{e}^{V^{-1}(u)} A^{\prime}\left(\mathrm{e}^{V^{-1}(u)}\right)}$ tends to 0 when $u$ tends to $s_{+}(F)$. As $\bar{G}_{0}$ is a decreasing function and $s \mapsto s+q_{u}(s)$ is an increasing one, we get

$$
\sup _{s \geq S(u)}\left|B_{u}(s)\right| \leq \bar{G}_{0}(S(u))+\bar{G}_{0}\left(S(u)+q_{u}(S(u))\right) \leq 2 \bar{G}_{0}(S(u))+\left|B_{u}(S(u))\right|
$$

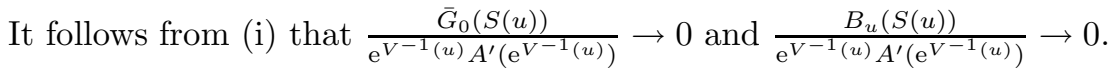

- In order to prove (i), we give the following lemmas:

Lemma 2. Let a be equal to 2 or 3.

For all $\epsilon>0$, we define on $\mathbb{R}_{+} \tilde{f}_{\gamma, \epsilon}$ and $\tilde{g}_{\gamma, \epsilon}$ by

$$
\begin{aligned}
& \tilde{f}_{\gamma, \epsilon}(x)=\left((1+\epsilon)^{a} \mathrm{e}^{2 \epsilon x}-1\right) M_{\gamma}(x) \mathrm{e}^{-(\gamma+1) x}, \\
& \tilde{g}_{\gamma, \epsilon}(x)=\left((1-\epsilon)^{a} \mathrm{e}^{-2 \epsilon x}-1\right) M_{\gamma}(x) \mathrm{e}^{-(\gamma+1) x} .
\end{aligned}
$$

If $\gamma>-1$,

$$
\limsup _{\epsilon \rightarrow 0}\left|\tilde{f}_{\gamma \geq 0}(x)\right|=0 \quad \text { and } \quad \lim _{\epsilon \rightarrow 0} \sup _{x \geq 0}\left|\tilde{g}_{\gamma, \epsilon}(x)\right|=0 .
$$

The proof of this lemma uses Lemma 3 and follows the same lines as the proof of Lemma 4 in [9] (or Lem. 4 in $[10])$.

Lemma 3. For any $\epsilon>0$,

if $s$ tends to $+\infty$, then, if $\gamma \geq 0, M_{\gamma}(s)=o\left(\mathrm{e}^{(\gamma+\epsilon) s}\right)$ and, if $\gamma<0, M_{\gamma}(s) \rightarrow-\frac{1}{\gamma^{3}}$;

if $s$ tends to $-\infty$, then, if $\gamma \geq 0, M_{\gamma}(s) \rightarrow-\frac{1}{\gamma^{3}}$ and, if $\gamma<0, M_{\gamma}(s)=o\left(\mathrm{e}^{(\gamma-\epsilon) s}\right)$.

If we let

$$
p_{u}(s)=\frac{V\left(s+V^{-1}(u)\right)-\alpha(u)}{\sigma(u)}-\frac{\mathrm{e}^{\lambda\left(V^{-1}(u)\right) s}-1}{\lambda\left(V^{-1}(u)\right)},
$$

we derive the following "Potter-type" bounds, consequences of Proposition 1.

Lemma 4. For all $\gamma \in \mathbb{R}$,

(i) $\forall \epsilon>0, \exists u_{0}, \forall u \geq u_{0}, \forall s \in \mathbb{R}_{+}$,

$$
(1-\epsilon) \mathrm{e}^{-\epsilon s} M_{\gamma}(s) \leq \frac{p_{u}(s)}{\lambda^{\prime}\left(V^{-1}(u)\right)} \leq(1+\epsilon) \mathrm{e}^{\epsilon s} M_{\gamma}(s) .
$$

(ii) $\lim _{u \rightarrow s_{+}(F)} p_{u}(s)=0$.

Remark 6. This lemma establishes that, for $u$ sufficiently large, $p_{u}(s)$ has the same sign as $\lambda^{\prime}\left(V^{-1}(u)\right)$ for all $s>0$.

$q_{u}$ (see (11)) and $p_{u}($ see $(15))$ are linked in the following way:

- if $\gamma=0, q_{u}(s)=p_{u}(s)$,

- if $\gamma \neq 0$ and $1+\Lambda(u) \frac{V\left(s+V^{-1}(u)\right)-\alpha(u)}{\sigma(u)}>0$ ( which is true for $u$ sufficiently large) there exists $\theta=\theta(u, s) \in$ $[0,1]$ such that

$$
q_{u}(s)=\mathrm{e}^{-\Lambda(u) s} p_{u}(s)\left(1+\theta \Lambda(u) \mathrm{e}^{-\Lambda(u) s} p_{u}(s)\right)^{-1} .
$$


The proof of the following lemma is similar to the proof of Lemma 6 in [9] (or Lem. 8 in [10]), the only difference being that $\gamma$ is replaced by $\Lambda(u)$, which tends to $\gamma$ as $u$ tends to $s_{+}(F)$.

Lemma 5. Let $S$ be a positive function defined on $\mathbb{R}_{+}$and satisfying $\lim _{u \rightarrow s_{+}(F)} S(u)=+\infty$.

(i) If $\gamma \geq 0$ and there exists $\epsilon>0$ such that

$$
\lim _{u \rightarrow s_{+}(F)} \ln \left|\lambda^{\prime}\left(V^{-1}(u)\right)\right|+3 \epsilon S(u)=-\infty
$$

for $u$ sufficiently large, $q_{u}(s)$ and $p_{u}(s)$ have the same sign and, as $u$ tends to $s_{+}(F)$,

$$
\sup _{s \in[0, S(u)]} \mathrm{e}^{-\Lambda(u) s}\left|p_{u}(s)\right| \longrightarrow 0 \text { and } \sup _{s \in[0, S(u)]}\left|q_{u}(s)\right| \longrightarrow 0 .
$$

(ii) If $\gamma<0$ and there exists $\epsilon>0$ such that

$$
\lim _{u \rightarrow s_{+}(F)} \ln \left|\lambda^{\prime}\left(V^{-1}(u)\right)\right|+(2 \epsilon-\gamma) S(u)=-\infty
$$

for $u$ sufficiently large, $q_{u}(s)$ and $p_{u}(s)$ have the same sign and, as $u$ tends to $s_{+}(F)$,

$$
\sup _{s \in[0, S(u)]} \mathrm{e}^{-\Lambda(u) s}\left|p_{u}(s)\right| \longrightarrow 0 \text { and } \sup _{s \in[0, S(u)]}\left|q_{u}(s)\right| \longrightarrow 0
$$

(iii) Condition (17) is fulfilled with $S(u)=-\alpha \ln \left|\lambda^{\prime}\left(V^{-1}(u)\right)\right|$, for $\alpha>0$ and condition (18) is fulfilled with $S(u)=-\alpha \ln \left|\lambda^{\prime}\left(V^{-1}(u)\right)\right|$, for $0<\alpha<-\frac{1}{\gamma}$.

- Here are the main steps of the proof of (i):

It is easy to check that if we choose (see Lem. 5 (iii))

$$
S(u)=-\alpha \ln \left|\mathrm{e}^{V^{-1}(u)} A^{\prime}\left(\mathrm{e}^{V^{-1}(u)}\right)\right|,
$$

with $\alpha>1$, then

$$
\frac{1}{\mathrm{e}^{V^{-1}(u)} A^{\prime}\left(\mathrm{e}^{V^{-1}(u)}\right)} \bar{G}_{0}(S(u)) \longrightarrow 0, \text { as } u \rightarrow s_{+}(F)
$$

Now, relation (16) and Lemma 5 yield bounds for $q_{u}(s)$ and then we deduce that there exists $u_{0} \in\left[0, s_{+}(F)[\right.$ such that, for $u \in\left[u_{0}, s_{+}(F)\left[\right.\right.$ and $s \in R_{+}$,

$$
(1-\epsilon)^{2} \frac{\mathrm{e}^{-\gamma s} p_{u}(s)\left|\bar{G}_{0}^{\prime}(s+\epsilon)\right|}{\mathrm{e}^{V^{-1}(u)} A^{\prime}\left(\mathrm{e}^{V^{-1}(u)}\right)} \leq \frac{B_{u}(s)}{\mathrm{e}^{V^{-1}(u)} A^{\prime}\left(\mathrm{e}^{V^{-1}(u)}\right)} \leq(1+\epsilon) \frac{\mathrm{e}^{-\gamma s} p_{u}(s)\left|\bar{G}_{0}^{\prime}(s)\right|}{\mathrm{e}^{V^{-1}(u)} A^{\prime}\left(\mathrm{e}^{V^{-1}(u)}\right)} .
$$

According to Lemma 4 , there exists $u_{1} \geq u_{0}$ such that, for $u \in\left[u_{1}, s_{+}(F)\left[\right.\right.$ and $s \in \mathbb{R}_{+}$,

$$
(1-\epsilon) \mathrm{e}^{-\epsilon s} M_{\gamma}(s) \leq \frac{p_{u}(s)}{\exp \left(V^{-1}(u)\right) A^{\prime}\left(\exp \left(V^{-1}(u)\right)\right)} \leq(1+\epsilon) \mathrm{e}^{\epsilon s} M_{\gamma}(s) .
$$

It follows from (19) and (20) that, for $u \in\left[u_{1}, s_{+}(F)[\right.$ and $s \in[0, S(u)]$,

$$
\left[(1-\epsilon)^{3} \mathrm{e}^{-\epsilon s}-1\right] \mathrm{e}^{-\gamma s} M_{\gamma}(s)\left|\bar{G}_{0}^{\prime}(s)\right| \leq \frac{B_{u}(s)}{\mathrm{e}^{V^{-1}(u)} A\left(\mathrm{e}^{V^{-1}(u)}\right)}-C_{\gamma}(s) \leq\left[(1+\epsilon)^{2} \mathrm{e}^{\epsilon s}-1\right] \mathrm{e}^{-\gamma s} M_{\gamma}(s)\left|\bar{G}_{0}^{\prime}(s)\right|
$$


The bounds in (21) being continuous functions tending to 0 when $\epsilon$ tends to 0 , the convergence of $\frac{B_{u}(s)}{\mathrm{e}^{V^{-1}(u)} A^{\prime}\left(\mathrm{e}^{V^{-1}(u)}\right)}$ towards $C_{\gamma}(s)$ is established on every compact set $[0, T](T>0)$.

We extend the convergence to $[0, S(u)]$ using Lemma 2.

\section{REFERENCES}

[1] A. Balkema and L. de Haan, Residual life time at great age. Ann. Probab. 2 (1974) 792-801.

[2] C.M. Goldie, N.H. Bingham and J.L. Teugels, Regular variation. Cambridge University Press (1987).

[3] J.P. Cohen, Convergence rates for the ultimate and penultimate approximations in extreme-value theory. Adv. Appl. Prob. 14 (1982) 833-854.

[4] R.A. Fisher and L.H.C. Tippet, Limiting forms of the frequency of the largest or smallest member of a sample. Proc. Cambridge Phil. Soc. 24 (1928) 180-190.

[5] M.I. Gomes, Penultimate limiting forms in extreme value theory. Ann. Inst. Stat. Math. 36 (1984) 71-85.

[6] I. Gomes and L. de Haan, Approximation by penultimate extreme value distributions. Extremes 2 (2000) 71-85.

[7] M.I. Gomes and D.D. Pestana, Non standard domains of attraction and rates of convergence. John Wiley \& Sons (1987) 467-477.

[8] J. Pickands III, Statistical inference using extreme order statistics. Ann. Stat. 3 (1975) 119-131.

[9] J.-P. Raoult and R. Worms, Rate of convergence for the Generalized Pareto approximation of the excesses (submitted).

[10] R. Worms, Vitesse de convergence de l'approximation de Pareto Généralisée de la loi des excès. Preprint Université de Marne-la-Vallée (10/2000).

[11] R. Worms, Vitesses de convergence pour l'approximation des queues de distributions Ph.D. Thesis Université de Marne-la-Vallée (2000). 